\title{
Colon cancer chemopreventive effects of baicalein, an active enteric microbiome metabolite from baicalin
}

\author{
CHONG-ZHI WANG ${ }^{1,2}$, CHUN-FENG ZHANG ${ }^{1-3}$, LINA CHEN ${ }^{1,2,4}$, SAMANTHA ANDERSON $^{1,2}$, \\ FANG LU ${ }^{1,2,5}$ and CHUN-SU YUAN ${ }^{1,2,6}$ \\ ${ }^{1}$ Tang Center for Herbal Medicine Research, ${ }^{2}$ Department of Anesthesia and Critical Care, University of Chicago, \\ Chicago, IL 60637, USA; ${ }^{3}$ State Key Laboratory of Natural Medicines, China Pharmaceutical University, \\ Nanjing, JS 210009; ${ }^{4}$ School of Pharmacy, Nanjing Medical University, Nanjing, JS 210029; \\ ${ }^{5}$ Chinese Medicine Toxicological Laboratory, Heilongjiang University of Chinese Medicine, Harbin, \\ HL 150040, P.R. China; ${ }^{6}$ Committee on Clinical Pharmacology and Pharmacogenomics, \\ University of Chicago, Chicago, IL 60637, USA
}

Received June 26, 2015; Accepted August 5, 2015

DOI: $10.3892 /$ ijo.2015.3173

\begin{abstract}
Baicalin is a major constituent of Scutellaria baicalensis, which is a commonly used herbal medicine in many Asian countries. After oral ingestion, intestinal microbiota metabolism may change parent compound's structure and its biological activities. However, whether baicalin can be metabolized by enteric microbiota and the related anticancer activity is not clear. In this study, using human enteric microbiome incubation and HPLC analysis, we observed that baicalin can be quickly converted to baicalein. We compared the antiproliferative effects of baicalin and baicalein using a panel of human cancer cell lines, including three human colorectal cancer (CRC) cell lines. In vitro antiproliferative effects on CRC cells were verified using an in vivo xenograft nude mouse model. Baicalin showed limited antiproliferative effects on some of these cancer cell lines. Baicalein, however, showed significant antiproliferative effects in all the tested cancer cell lines, especially on HCT-116 human colorectal cancer cells. In vivo antitumor results supported our in vitro data. We demonstrated that baicalein exerts potent $\mathrm{S}$ phase cell cycle arrest and pro-apoptotic effects in HCT-116 cells. Baicalein induced the activation of caspase 3 and 9. The in silico modeling suggested that baicalein forms hydrogen bonds with residues Ser251 and Asp253 at the active site of caspase 3, while interactions with residues Leu227 and Asp228 in caspase 9 through its hydroxyl groups. Data from this study
\end{abstract}

Correspondence to: Dr Chong-Zhi Wang, Tang Center for Herbal Medicine Research, University of Chicago, 5841 S. Maryland Ave., MC 4028, Chicago, IL 60637, USA

E-mail: cwang@dacc.uchicago.edu

Key words: baicalein, baicalin, Scutellaria baicalensis, enteric microbiome metabolite, anticancer, cell cycle, caspases, apoptosis, receptor-ligand docking, colorectal cancer suggested that baicalein is a potent anticancer metabolite derived from S. baicalensis. Enteric microbiota play a key role in the colon cancer chemoprevention of S. baicalensis.

\section{Introduction}

Colorectal cancer (CRC) is one of the most common cancers worldwide $(1,2)$. In the United States, it is expected that there will be 132,700 newly diagnosed CRC cases and 49,700 CRC-related deaths in 2015 (2), indicating the inadequacies of currently available measures $(1,3)$. Herbal medication is an approach that has recently gained more attention for colorectal cancer (CRC) management $(4,5)$. It is known that botanicals have been a significant resource to several of the currently used efficacious chemotherapeutic agents $(6,7)$. The identification of non-toxic natural compounds from herbal medicines remains an essential step in advancing CRC therapeutics $(8,9)$.

The root of Scutellaria baicalensis is a widely used herbal medicine in the traditional medical systems of China and Japan for a variety of inflammation related ailments (10-13). The major constituents of this botanical are a group of flavonoid glycosides, including baicalin, and wogonoside, of which baicalin is the major constituent in the herb $(14,15)$.

S. baicalensis is most often orally administered. After oral ingestion, the constituents in the herb inevitably come into contact with intestinal microbiota. Many of these constituents could be transformed by the intestinal bacteria before being absorbed (16). As reported before, for natural glycosides such as ginsenosides, the most common metabolic pathway is the deglycosylation reaction induced by intestinal bacteria via the stepwise cleavage of the sugar moieties (17-19). After deglycosylation, compared to their parent compounds, the intestinal microbiome metabolites may have more potent biological activity (20-22).

Anticancer activities of S. baicalensis and its constituents were reported, but previous studies focused more on its natural sourced flavonoid glycosides $(23,24)$. We recently observed that the major constituent of $S$. baicalensis, baicalin, can be 
converted to baicalein by glycoside hydrolases or hydrolyzing during the herb's processing or storage $(20,25)$, but whether the enteric microbiome will metabolize baicalin is still unclear. In addition, although attempts have been made to evaluate the anticancer activities of the two compounds $(26,27)$, a chemopreventive effect comparison between baicalin and baicalein on CRC has not been performed.

In this study, using the human microbiome, we determined biotransformation from baicalin to baicalein. We compared the antiproliferative effects of baicalin and baicalein using a panel of human cancer cell lines, including three human CRC cell lines. The in vitro antiproliferative effects on CRC cells were verified using an in vivo xenograft nude mouse model. Then, we selected HCT-116 colon cancer cells, which are most sensitive to baicalein treatment, for further mechanistic observations, including cell cycle arrest and apoptosis induction. Due to the fact that caspases are highly conserved in multicellular organisms and function as central regulators of apoptosis, levels of caspase expression were subsequently determined. Finally, the possible binding modes of baicalein at the catalytic domains of caspase 3 and 9 were simulated using the receptor-ligand docking analysis.

\section{Materials and methods}

Chemicals and materials. All cell culture plasticware were obtained from Falcon Labware (Franklin Lakes, NJ, USA) and Techno Plastic Products (Trasadingen, Switzerland). Trypsin, McCoy's 5A, Leibovitz's L-15, RPMI-1640 and DMEM media, and phosphate-buffered saline were obtained from Mediatech, Inc. (Herndon, VA, USA). Penicillin and streptomycin were obtained from Sigma-Aldrich (St. Louis, MO, USA). The MTS assay kit, CellTiter 96 Aqueous Solution Cell Proliferation Assay, was obtained from Promega (Madison, WI, USA). The Annexin V-FITC apoptosis detection kit was obtained from BD Biosciences (Rockville, MD, USA). PI/RNase staining buffer was obtained from BD Biosciences Pharmingen (San Diego, CA, USA). Caspase 3 and 9 ELISA kits were obtained from BioVison (Mountain View, CA, USA). Baicalin and wogonoside were obtained from Indofine Chemical Co. Inc. (Hillsborough Township, NJ, USA). Baicalein and wogonin were obtained from Sigma-Aldrich.

Plant materials and extraction. The roots of Scutellaria baicalensis were obtained from Chengde (Hebei, China). The voucher samples were deposited at the Tang Center for Herbal Medicine Research at The University of Chicago. Dried S. baicalensis roots were ground to powder, and the powdered roots were extracted with $70 \%$ ethanol for $2 \mathrm{~h}$. The extraction method was boiling under reflux. The filtrate was collected and the extraction procedure was repeated one more time on the residue. The combined filtrate was condensed under vacuum and lyophilized to yield dried S. baicalensis extract (SbE).

Biotransformation of SbE by human fecal microflora. Fecal samples were obtained from five adult volunteers, who were non-smokers and had not consumed antibiotics for $\geq 3$ months before the study. The samples were collected by the donors in plastic cups, and were processed within $30 \mathrm{~min}$ of passage. All five fecal samples were mixed and an aliquot of $5 \mathrm{~g}$ of the mixed feces was homogenized with $20 \mathrm{ml}$ of phosphate buffer ( $\mathrm{pH}$ 7.0) to obtain a fecal slurry. The slurry was filtered through muslin to remove particulate material. One microliter of the fecal slurry was mixed with $4 \mathrm{ml}$ anaerobic medium containing $2.5 \mathrm{mg}$ of SbE. They were anaerobically incubated at $37^{\circ} \mathrm{C}$ for 0,2 or $8 \mathrm{~h}$. Then, $1 \mathrm{ml}$ of reaction mixture was extracted three times with $400 \mu \mathrm{l}$-butanol/each time. The combined n-butanol solution was dried under nitrogen steam spray in a water bath $\left(60^{\circ} \mathrm{C}\right)$. Then the residue was dissolved in methanol. The methanol solution was centrifuged at $17,000 \mathrm{x} \mathrm{g}$ for $10 \mathrm{~min}$ before HPLC analysis.

High performance liquid chromatography (HPLC) analysis. The HPLC system was a Waters 2960 instrument (Milford, MA, USA) with a quaternary pump, an automatic injector, a photodiode array detector (Model 996), and Waters Empower software for peak identification and integration. The separations were carried out on a Phenomenex Prodigy ODS(2) column $(150 \times 2.0 \mathrm{~mm}, 5 \mu \mathrm{m})$. A binary gradient solvent system of acetonitrile (eluent $\mathrm{A})-0.03 \%(\mathrm{v} / \mathrm{v})$ phosphoric acid in water (eluent B) was used as follows: $13 \% \mathrm{~A}$ and $87 \% \mathrm{~B}$ (0 min), $28 \% \mathrm{~A}$ and $72 \% \mathrm{~B}$ (17 $\mathrm{min}), 35 \% \mathrm{~A}$ and $65 \% \mathrm{~B}(27 \mathrm{~min})$, $90 \% \mathrm{~A}$ and $10 \% \mathrm{~B}(30-31 \mathrm{~min}), 13 \% \mathrm{~A}$ and $87 \% \mathrm{~B}$ (34-39 min). The flow-rate of $0.8 \mathrm{ml} / \mathrm{min}$ was used and absorbance was detected at $280 \mathrm{~nm}$. All tested solutions were filtered through Millex $0.2-\mu \mathrm{m}$ nylon membrane syringe filters before use. The contents of the constituents were calculated using standard curves of flavonoids.

Cell lines and cultures. The human colorectal cancer cell lines HCT-116 (McCoy's 5A), SW-480 (Leibovitz's L-15), HT-29 (McCoy's 5A), NSCLC non-small cell lung cancer cells (DMEM), and human breast cancer cell lines MCF-7 (RPMI-1640), MDA-MB-231 (RPMI-1640) were obtained from American Type Culture Collection (Manassas, VA, USA). The cells were grown in the indicated medium supplemented with $10 \%$ FBS and 50 IU penicillin/streptomycin in a humidified atmosphere with $5 \% \mathrm{CO}_{2}$ at $37^{\circ} \mathrm{C}$.

Cell proliferation analysis. Baicalin and baicalein were dissolved in DMSO and were stored at $-20^{\circ} \mathrm{C}$ before use. Cells were seeded in 96-well plates $\left(1 \times 10^{4}\right.$ cells/well). After $24 \mathrm{~h}$, indicated concentrations of drugs were added to the wells. The final concentration of DMSO was $1 \%$. Controls were exposed to culture medium containing 1\% DMSO without drugs. All experiments were performed in triplicate and repeated 3 times. Following the indicated incubation period, cell proliferation was evaluated using an MTS assay according to the manufacturer's instructions. Briefly, the medium was replaced with $100 \mu \mathrm{l}$ of fresh medium and $20 \mu \mathrm{l}$ of MTS reagent (CellTiter 96 Aqueous Solution) in each well, and the plate was returned to the incubator for 1-2 h. A $60-\mu 1$ aliquot of medium from each well was transferred to an ELISA 96-well plate and its absorbance at $490 \mathrm{~nm}$ was recorded. Since 1\% DMSO did not influence the proliferation of the two cell lines, results were expressed as percent of control (DMSO vehicle set at 100\%).

Cell cycle analysis. HCT-116 cells were seeded in 24-well tissue culture plates. On the second day, the medium was changed and cells were treated with test compounds. Cells 
were incubated for $48 \mathrm{~h}$ before they were harvested. These cells were fixed gently with $80 \%$ ethanol in a freezer for $2 \mathrm{~h}$ and were then treated with $0.25 \%$ Triton X-100 for 5 min on an ice bath. Cells were resuspended in $300 \mu \mathrm{l}$ of PBS containing $40 \mu \mathrm{g} / \mathrm{ml}$ propidium iodide (PI) and $0.1 \mathrm{mg} / \mathrm{ml}$ RNase (28). Then the cells were incubated in the dark for $20 \mathrm{~min}$ at room temperature, and cell cycle analysis was performed using a FACScan flow cytometer (Becton-Dickinson, Mountain View, CA, USA) and FlowJo 7.1.0 software (Tree Star, Ashland, OR, USA). For each measurement, $\geq 10,000$ cells were counted.

Apoptotic analysis. The apoptosis assay was performed by flow cytometry following a previously described procedure (29). Briefly, HCT-116 cells were seeded in 24-well tissue culture plates. After culturing for 1 day, the medium was changed and test compounds were added. After treatment for $48 \mathrm{~h}$, cells floating in the medium were collected. The adherent cells were detached with trypsin. Then, culture medium containing $10 \%$ FBS (and floating cells) was added to inactivate trypsin. After gentle pipetting, the cells were centrifuged for $5 \mathrm{~min}$ at 1,500 g. The supernatant was removed and cells were stained with Annexin V-fluorescein isothiocyanate (FITC) and propidium iodide (PI) according to the manufacturer's instructions. Untreated cells served as controls. The cells were analyzed immediately after staining using a flow cytometer. For each measurement, $\geq 20,000$ cells were counted.

Caspase 3 and 9 analyses. HCT-116 cells were seeded in 6 -well tissue culture plates. After $24 \mathrm{~h}$, the medium was changed and baicalein was added. After treatment for $24 \mathrm{~h}$, cell lysates were collected. Expression levels of caspase 3 and 9 were determined by the colorimetric method according to the manufacturer's instructions. Briefly, cell lysates were diluted with $50 \mu \mathrm{l}$ of $2 \mathrm{X}$ reaction buffer (containing $10 \mathrm{mM}$ DTT) to a protein concentration of $0.5 \mathrm{mg} / \mathrm{ml}$ in an ELISA 96-well plate. Then, $5 \mu 1$ of colorimetric tetrapeptide substrate (DEVD-pNA for caspase 3 and LEHDpNA for caspase 9) and cell lysate were added, and plate was incubated at $37^{\circ} \mathrm{C}$ for $24 \mathrm{~h}$. Then, the absorbance was recorded at $405 \mathrm{~nm}$. The change in caspase activity was calculated as absorbance of baicalein treated cells/absorbance of untreated controls.

Receptor docking analysis. The possible binding modes of baicalein at the catalytic domains of human caspase 3 and caspase 9 were predicted using the docking program SurflexDock (Tripos, St. Louis, MO, USA). The structure of baicalein was generated (through Ligand model in Sybyl), and protein crystal structures were obtained (PDB code $3 \mathrm{HOE}$ for caspase 3 and 2AR9 for caspase 9). To prepare for docking analysis, the protein structures were prepared by adding hydrogen atoms and missing sidechain atoms and removing water molecules. Intermolecular interaction between baicalein and caspases were analyzed, and the key pharmacophore in the ligand was identified $(30,31)$.

Statistical analysis. Data are presented as mean \pm standard error (SE) (n=3). A one-way ANOVA was employed to determine the statistical significance of the results. When necessary, a Student's t-test was used to compare the two groups. The level of statistical significance was set at $\mathrm{P}<0.05$.

\section{Results}

Baicalin metabolism by human enteric microbiome to produce baicalein. HPLC analysis was used to monitor SbE flavonoid changes during the biotransformation of human enteric microbiome. As shown in Fig. 1, four flavonoids were detected in SbE, i.e., baicalin, wogonoside, baicalein and wogonin. Baicalin is the major constituent in SbE. To determine whether fecal compounds influence SbE flavonoid analysis, we assayed the vehicle fecal sample. A major peak at retention time (Rt) of $9.061 \mathrm{~min}$ was observed in the chromatogram of the fecal sample. For the $\mathrm{SbE}$, the closest flavonoid peak to this fecal peak is baicalin, with an Rt of 11.366 min. This fecal peak was separated from the baicalin peak at baseline. Thus, compounds from fecal sample did not influence SbE flavonoid determination.

When SbE was cultured with human enteric microbiome for $2 \mathrm{~h}$, compared to un-transformed $\mathrm{SbE}$, the baicalin peak was significantly reduced. Data showed that $75.3 \%$ of baicalin in $\mathrm{SbE}$ was converted to baicalein after being cultured for $2 \mathrm{~h}$. Furthermore, after being cultured for $8 \mathrm{~h}$, baicalin was not detected, while baicalein was the major constituent in the reaction mixture, indicating that all baicalin in SbE was converted to baicalein. Similar results were also observed in wogonoside. Our data showed that the gut microbiome can quickly transform baicalin to baicalein.

Antiproliferative effects of baicalin and baicalein. In this study, six human cancer cell lines from three of the most common human cancers were used, including colorectal cancer (HCT-116, SW-480 and HT-29), non-small cell lung cancer (NSCLC), and breast cancer (MCF-7 and MDA-MB-231).

As shown in Fig. 2A-C, while 48-h treatment with baicalin inhibited cancer cell growth in relatively high concentrations, baicalein caused much stronger growth suppression in all three colorectal cancer cell lines. At $60 \mu \mathrm{M}$, only baicalin showed $7.6 \pm 1.7 \%$ of an antiproliferative effect on HCT-116 cells $(\mathrm{P}<0.05$ vs. control), while no significant effects were observed on the other two cancer cell lines. In the same concentration $(60 \mu \mathrm{M})$, baicalein inhibited cancer cell growth by $73.7 \pm 3.4 \%$ in HCT-116, 40.3 $\pm 2.9 \%$ in SW-480, and $22.3 \pm 1.8 \%$ in HT-29 cells, respectively (all $\mathrm{P}<0.01$ vs. control). Among the three cell lines, baicalein showed the most potent antiproliferative effects in HCT-116 cells with an $\mathrm{IC}_{50}$ value of $40.1 \mu \mathrm{M}$.

Baicalein also showed significant antiproliferative effects on the other three cancer cell lines, but NSCLC cells were not sensitive to baicalein treatment. Compared to baicalein, baicalin showed weaker antiproliferative effects on NSCLC and MDA-MB-231 cells (Fig. 2D and F). MCF-7 cells were the only exception. Although baicalein showed significant effects on MCF-7 cells at concentrations even as low as $20 \mu \mathrm{M}$, when treatment concentration was increased to $100 \mu \mathrm{M}$, baicalin still did not inhibit cancer cell growth (Fig. 2E).

Our data suggested that baicalin showed less antiproliferative effects in the tested concentrations. Its enteric microbiome metabolite, baicalein, showed very significant antiproliferative effects in different human cancer cell lines, especially HCT-116 cells (Fig. 2).

Antitumor effects of baicalin and baicalein in xenograft tumor model. To confirm the in vitro antiproliferative effect 
<smiles>O=C1CC(c2ccccc2)Oc2cc(OC3OC(C(=O)O)C4OC3C(O)C4O)c(O)c(O)c21</smiles>

Baicalin<smiles>CC=CC1CC(=O)c2c(cc(O)c(O)c2O)O1</smiles>

Baicalejn
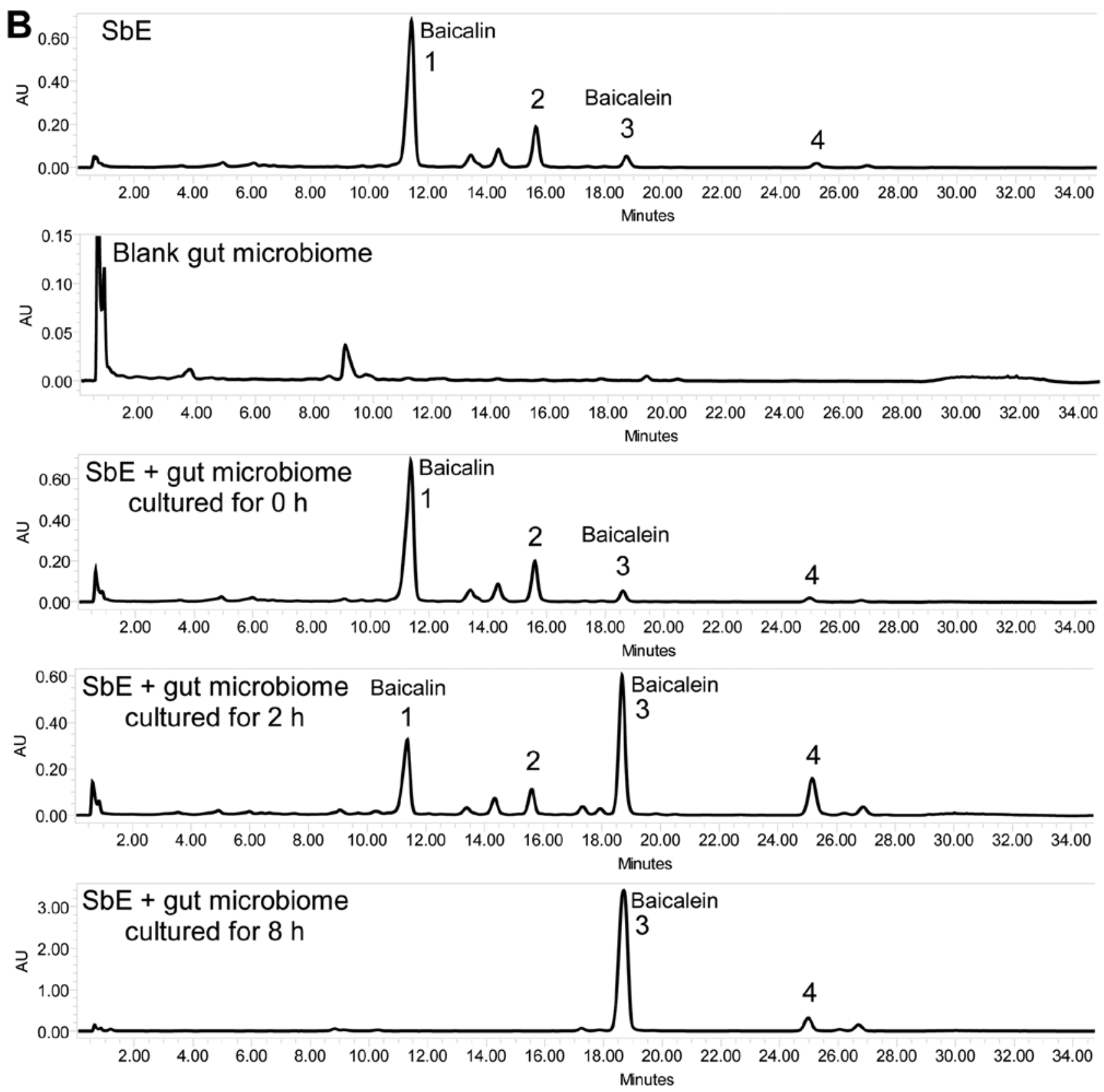

Figure 1. Biotransformation of S. baicalensis extract (SbE) by the human enteric microbiome. (A) Chemical structures of baicalin and baicalein. (B) HPLC analysis of $S$. baicalensis flavonoids transformed by enteric microbiome. Chromatograms of SbE, gut microbiome, SbE + gut microbiome, without culture $(0 \mathrm{~h})$, or cultured at $37^{\circ} \mathrm{C}$ for 2 and $8 \mathrm{~h}$ are shown. Peak number: 1, baicalin; 2, wogonoside; 3, baicalein; 4, wogonin.

of baicalein on HCT-116 colorectal cancer cells, the in vivo antitumor activities of baicalin and baicalein were evaluated. Firefly luciferase-tagged HCT-116 cells were inoculated into the flanks of athymic nude mice. Beginning on day 1 , animals were also administered with baicalin or baicalein at
$30 \mathrm{mg} / \mathrm{kg}$ or vehicle intraperitoneally every other day. Tumor growth was measured by xenogeny bioluminescence imaging on a weekly basis. Representative xenogen imaging results at weeks 0-4 are shown in Fig. 3A. Tumor size at indicated timepoints as assessed by imaging signal intensities is summarized 
A

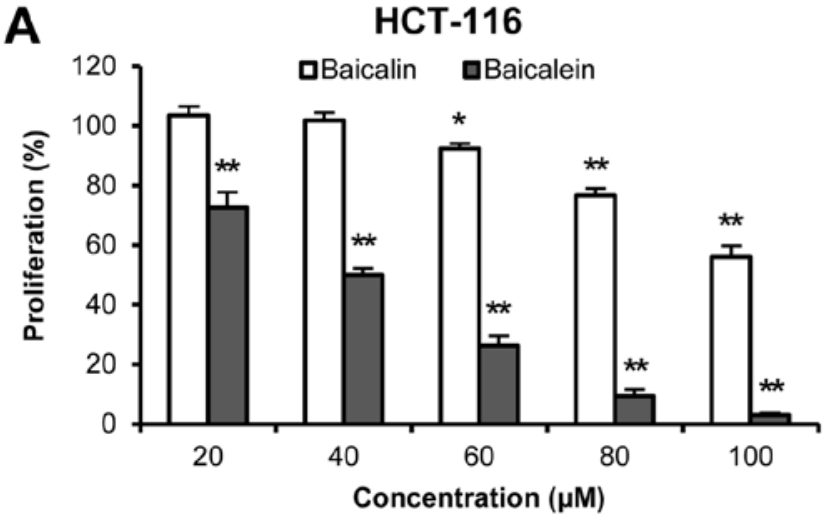

B

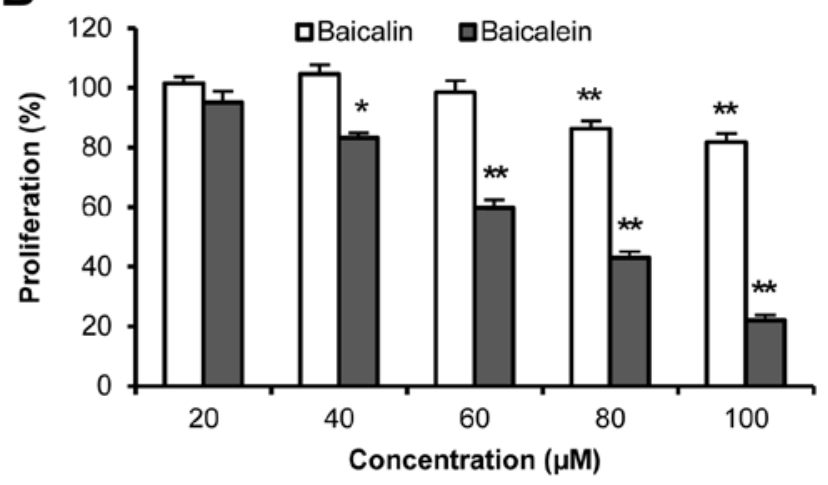

D

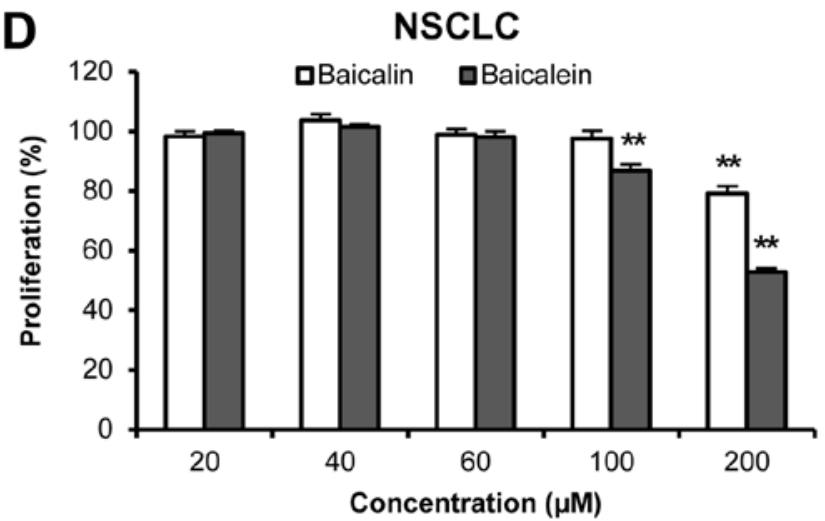

F

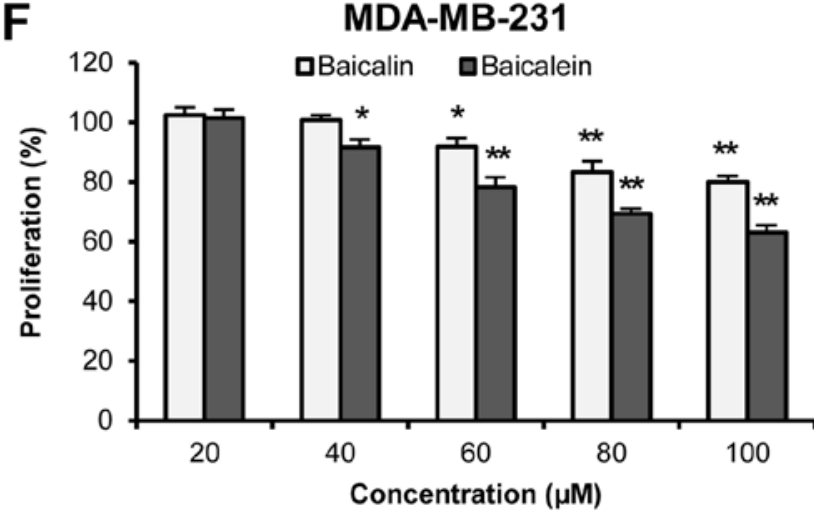

Figure 2. Effects of baicalin and baicalein on the proliferation of different human cancer cell lines assayed by the MTS method. Cell lines used include colorectal cancer (SW-480, HCT-116, HT-29), non-small cell lung cancer cells (NSCLC) and breast cancer (MCF-7, MDA-MB-231). Cells were treated with $10-200 \mu \mathrm{M}$ of tested compounds for $48 \mathrm{~h}$. ${ }^{*} \mathrm{P}<0.05$; and ${ }^{* *} \mathrm{P}<0.01$ vs. control $(100 \%)$.

in Fig. 3B. The data showed that at weeks 2 and 3, baicalin suppressed tumor growth, but there is no significant differences compared to control. At week 4, baicalin significantly inhibited tumor growth $(\mathrm{P}<0.05)$. For the baicalein, at week 2 , baicalein exhibited significantly decreased xenogeny imaging signal intensities compared with those of the control $(\mathrm{P}<0.05)$. Weeks 3 and 4 exhibited more significant antitumor effects than week 2 (both $\mathrm{P}<0.01$ ). Our data suggested the metabolite baicalein showed more significant antitumor effects than those of its parent compound baicalin. The enteric microbiome metabolism plays an important role in enhancing the anticancer activity of $\mathrm{SbE}$.

Effects of baicalin and baicalein on cell cycle. Since HCT-116 colorectal cancer cells were sensitive to baicalein treatment both in vitro and in vivo, we selected this cell line for further mechanistic evaluations. As shown in Fig. 4, compared to the control, the effects of baicalein on the cell cycle profile were observed at concentrations as low as $20 \mu \mathrm{M}$. Treatment of HCT-116 cells with 20, 40 and $60 \mu \mathrm{M}$ baicalein for $48 \mathrm{~h}$ decreased G1 phase to $25.0,19.0$ and $26.7 \%$, respectively, compared to $41.8 \%$ in vehicle treated cells, while increasing $\mathrm{S}$ phase to $49.7,60.7$ and $73.6 \%$, respectively, compared to $43.3 \%$ in vehicle treated cells (all $\mathrm{P}<0.01$ ). Thus, baicalein significantly decreased the number of cancer cells in G1 phase and increased the number of cancer cells in $S$ phase. On the other hand, baicalin treatment did not influence the cell cycle at 20 and $40 \mu \mathrm{M}$. Treatment with $60 \mu \mathrm{M}$ of baicalin for $48 \mathrm{~h}$ changed cell proportions by increasing G1 phase and decreasing $\mathrm{G} 2 / \mathrm{M}$ phase ( $\mathrm{P}<0.05$ vs control). These results 

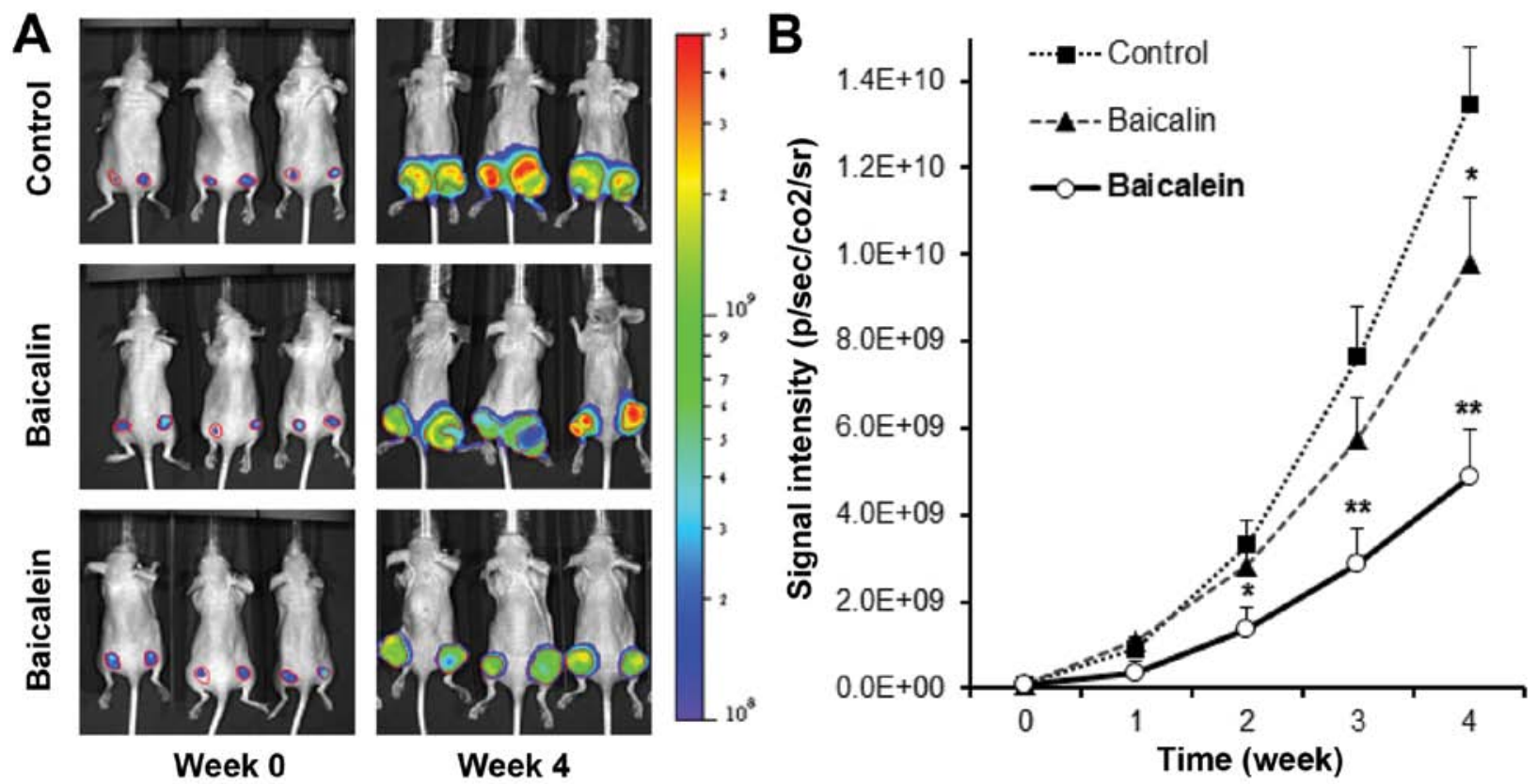

Figure 3. In vivo antitumor activity of baicalein in a xenograft mouse model. Firefly luciferase-tagged HCT-116 cells were injected into both flanks of athymic mice subcutaneously ( $\mathrm{n}=10 / \mathrm{group}$ ), and the tumor sizes after treatment with solvent control or $30 \mathrm{mg} / \mathrm{kg} / \mathrm{day}$ of baicalein were measured on a weekly basis by xenogen bioluminescence imaging. (A) Representative xenogen imaging results are shown. (B) Quantitative analysis of xenogen bioluminescence imaging. Average tumor sizes at the indicated time-points are represented with imaging signal intensities (in photons $/ \mathrm{second} / \mathrm{cm}^{2} / \mathrm{steradian)}$ ) as mean \pm standard error. ${ }^{*} \mathrm{P}<0.05,{ }^{* *} \mathrm{P}<0.01$ vs. control.

A
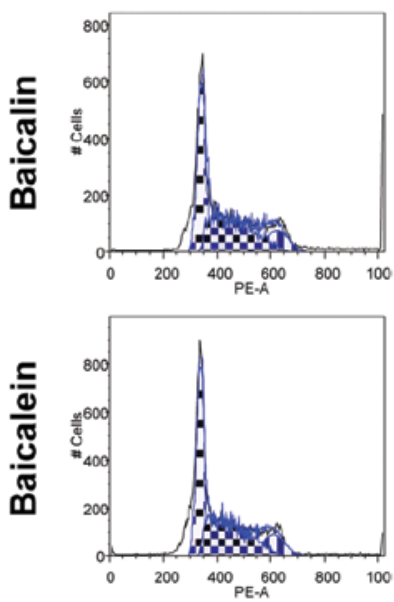

Control
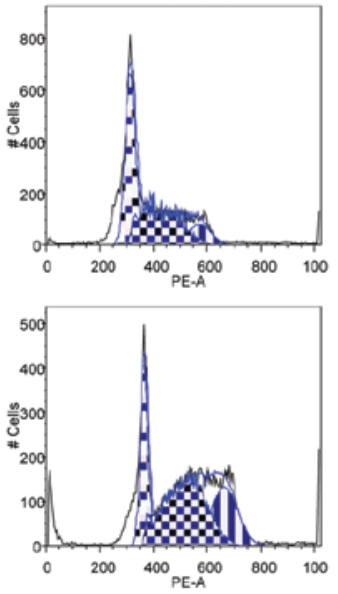

$20 \mu \mathrm{M}$
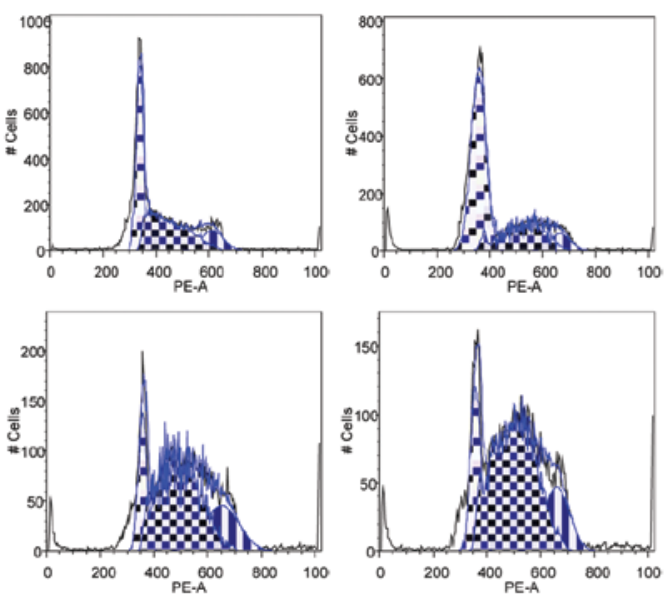

$40 \mu \mathrm{M}$

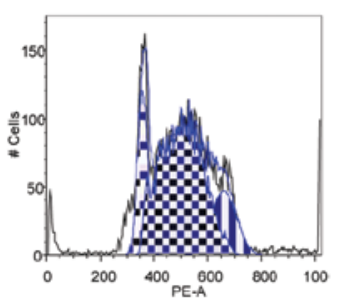

$60 \mu \mathrm{M}$
B

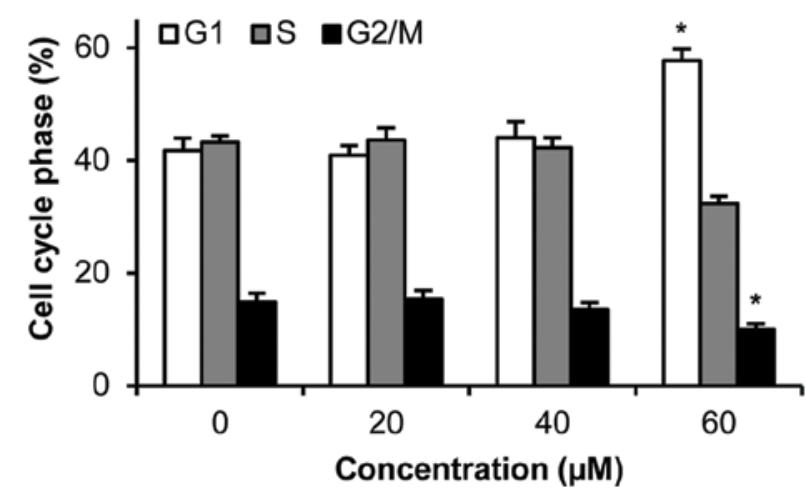

Baicalin 


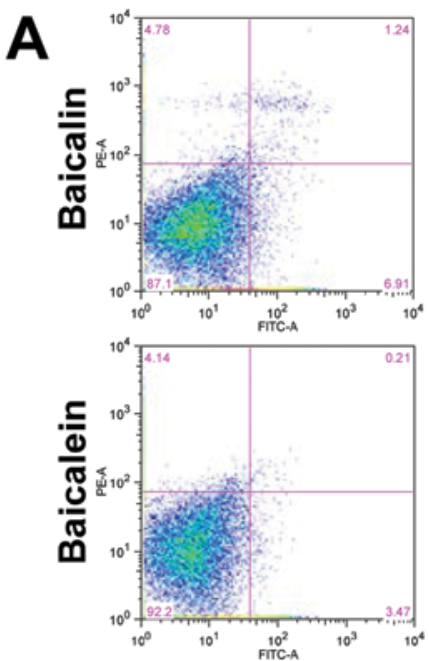

Control
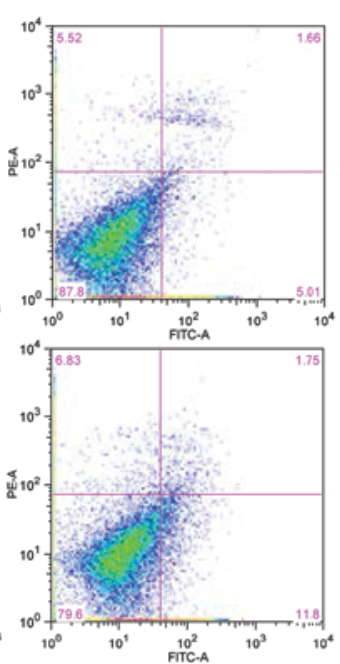

$20 \mu \mathrm{M}$
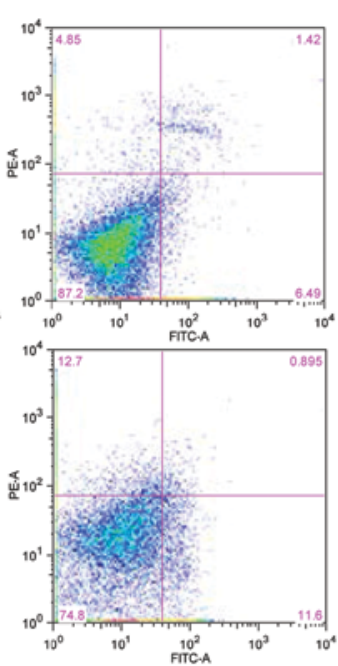

$40 \mu \mathrm{M}$
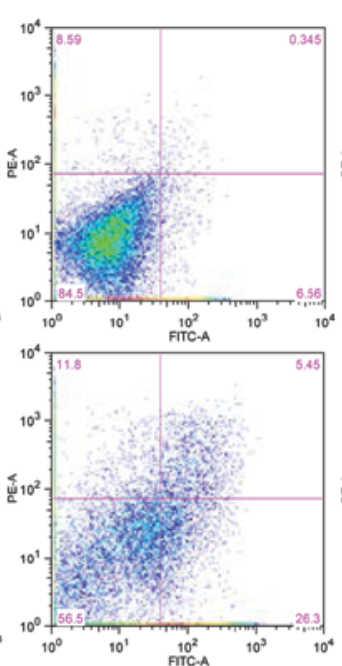

$60 \mu \mathrm{M}$
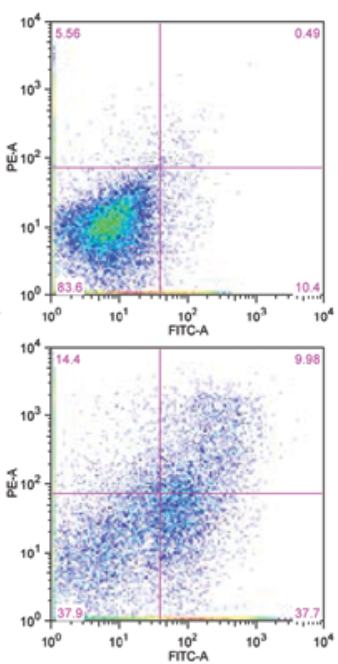

$80 \mu \mathrm{M}$
B

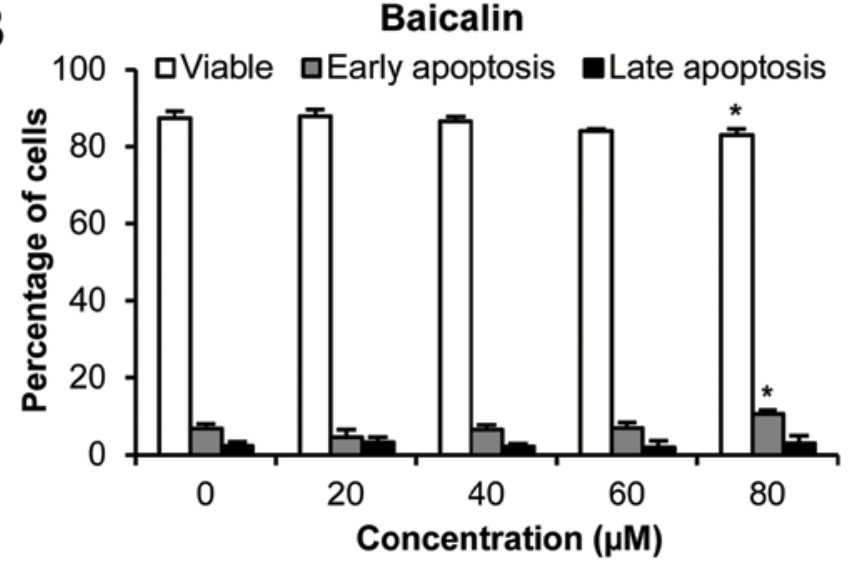

Baicalein

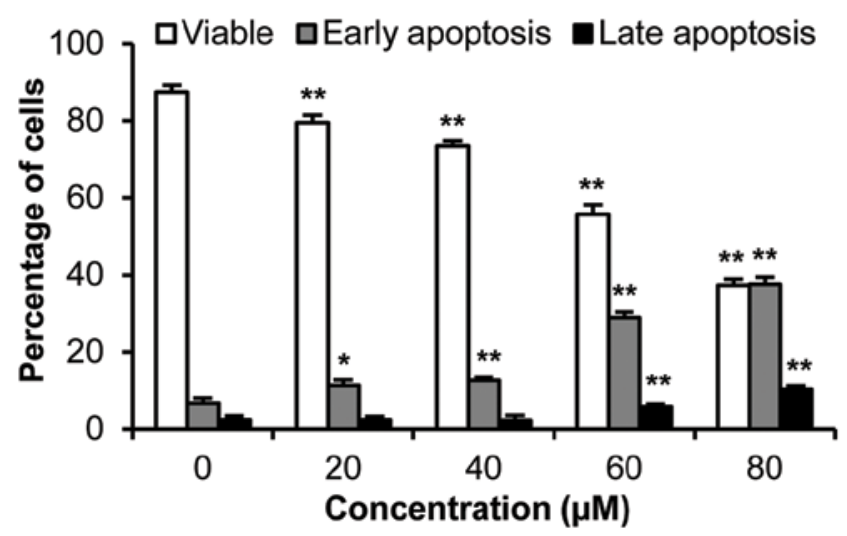

Figure 5. Apoptosis assay using flow cytometry after staining with Annexin V-FITC/propidium iodide (PI). HCT-116 cells were treated with $20-80 \mu \mathrm{M}$ of baicalin or baicalein for 48 h. (A) Representative scatter plots of PI (y-axis) vs. Annexin V (x-axis). (B) Percentage of viable, early apoptotic and late apoptotic cells. Data are presented as the means \pm SE of triplicate experiments. ${ }^{*} \mathrm{P}<0.05$, and ${ }^{* *} \mathrm{P}<0.01$ vs. control.

suggested that the metabolite baicalein, not parent compound baicalin, significantly induced $S$ phase cell cycle arrest in HCT-116 cells.

Effects of baicalin and baicalein on apoptosis. The apoptotic effects of baicalin and baicalein were evaluated by flow cytometry after staining with Annexin V and PI. Annexin V can be detected in both early and late stages of apoptosis, whereas PI stained cells only in late apoptosis or necrosis. Early apoptotic cells were positive for Annexin V and negative for PI (lower right quadrant); late apoptotic cells stained for both Annexin V and PI (upper right quadrant). As shown in Fig. 5, following treatment with $20,40,60$ and $80 \mu \mathrm{M}$ of baicalein for $48 \mathrm{~h}$, compared to the control $(6.7 \%)$, the percentage of early apoptotic SW-480 cells was increased to $11.2,12.5,28.8$ and $37.5 \%$, respectively $(\mathrm{P}<0.05, \mathrm{P}<0.01, \mathrm{P}<0.01$ and $\mathrm{P}<0.01)$. However, baicalin did not induce apoptosis at the concentrations of 20-60 $\mu \mathrm{M}$. Only $80 \mu \mathrm{M}$ of baicalin showed an antiproliferative effect, which induced $10.5 \%$ of early apoptotic cells $(\mathrm{P}<0.05)$. These data demonstrate that baicalein significantly induces cell apoptosis.
Effects baicalein on activities of caspase 3 and 9. Caspase 3 and caspase 9 are two key proteins of the caspase family of proteases, which are highly conserved in multicellular organisms and function as central regulators of apoptosis. They have been identified as playing a key role in the progression of apoptosis (32). To characterize the potential mechanism of baicalein's anticancer activity, we assayed the activities of two caspases since baicalein increased cancer cell apoptosis. As shown in Fig. 6A, treatment of HCT-116 cells with $40 \mu \mathrm{M}$ baicalein for 24 h upregulated caspase 3 and 9 activities significantly. These activities were further enhanced with $60 \mu \mathrm{M}$ of baicalein, increasing caspase 3 and 9 activities to $103.7 \pm 18.5$ and $95.4 \pm 16.4 \%$ above those of vehicle treated cells, respectively (both $\mathrm{P}<0.01$ ). Our results suggested that baicalein significantly induced the expression of caspase 3 and 9.

Molecular modeling of caspase 3 and 9 and the binding mode of baicalein. To further explore the potential effects of baicalein on caspase 3 and 9, we performed docking analysis to characterize the physical interactions of baicalein with these caspases. We examined baicalein docking for human 

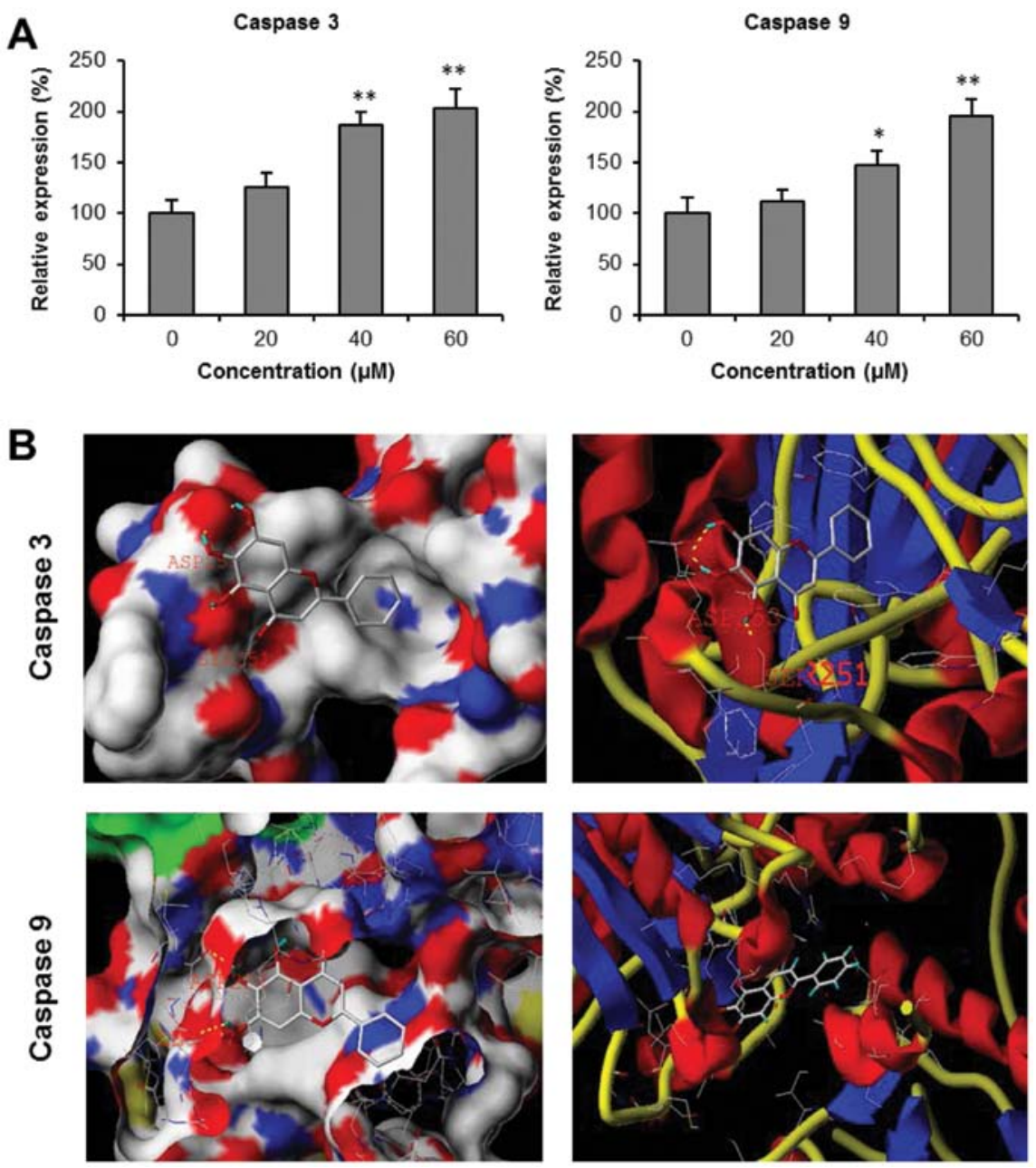

Figure 6. Effects of baicalein on caspase 3 and 9 activities in HCT-116 cells and docking analysis. (A) After treatment with $20-60 \mu \mathrm{M}$ of baicalein for $24 \mathrm{~h}$, cell lysates were prepared and enzymatic activities were measured by colorimetric assay for caspase 3 and 9 . Results are normalized to each control in percentage and expressed as the means \pm SE of triplicate experiments. $\mathrm{P}<0.05$, and ${ }^{* *} \mathrm{P}<0.01$ vs. control. (B) Three-dimensional docking model of baicalein at the binding site of human caspase 3 and caspase 9 proteins. The possible binding modes of baicalein at the catalytic domains of caspases were predicted using the docking program Surflex-Dock. The surface views are shown in the left panel, and stick-ribbon models on the right.

caspase 3 (PDB code: 3H0E) and human caspase 9 (PDB code: 2AR9). The Surflex-Dock program was used to predict the binding sites of baicalein to caspase 3 and 9 . The energetically most favorable positions for baicalein interaction with these caspases are shown in Fig. 6B. The in silico modeling suggested that baicalein forms hydrogen bonds with residues Ser251 and Asp253 at the active site of caspase 3, while baicalein forms hydrogen bonds with residues Leu227 and Asp228 in caspase 9 through its hydroxyl groups. In addition, baicalein is predicted to show significant binding affinity for caspase 3 (CScore 3.76) and caspase 9 (CScore 3.18), suggesting that baicalein may directly interact with these caspases.

\section{Discussion}

Colorectal cancer is the second leading cause of cancer related death in the United States, and the second most prevalent cancer worldwide $(1,2)$. The clinical CRC management involves diverse conventional modalities, including surgery, radiation, and chemotherapy $(2,33)$. The complex characteristics of human cancer also require some complementary approaches, including herbal medication, to improve the therapeutic efficacy of conventional therapies $(34,35)$. In the past 30 years, nearly $80 \%$ of approved anticancer drugs were derived from natural compounds (6). Herbal medicine has contributed significantly to CRC therapies and many of the novel compounds with significant anticancer properties are likely to be found in plant sources.

S. baicalensis is one of the most commonly used herbs in traditional medicine for the treatment of various inflammatory diseases in Asia. The representative constituents of S. baicalensis are a group of flavonoids that include glycosides (baicalin, wogonoside) and their aglycon metabolites (baicalein and wogonin), while baicalin occupies the major content of the total flavonoids $(14,15)$. In recent years, the anticancer activities of S. baicalensis extract and its constituents were reported $(36,37)$. However, most studies focused on its glycosides, such as baicalin, which possess only limited anticancer activities.

S. baicalensis is most often orally administered. In natural products research, many previous studies employed primarily reductionist approaches in screening compounds for bioactivity, and often only parent compounds were investigated. In fact, in the gut, parent flavonoid glycosides in S. baicalensis could be metabolized by the gut microbiome. However, whether 
baicalin can be metabolized by the enteric microbiome and its microbial metabolite leads to modified anti-colorectal cancer activities are largely unclear.

In this study, we investigated the biotransformation of baicalin by the human enteric microbiome. In our pilot study, we selected 2, 8 and 24-h incubation periods, based on our previous ginseng research (25), and observed $8 \mathrm{~h}$ was enough to allow for the full metabolism of the parent compounds. In this study, we observed that after $2 \mathrm{~h}$ of incubation time, $3 / 4$ of baicalin was converted to baicalein. After 8-h incubation, all baicalin had been metabolized to baicalein. Similar results were also observed on another pair of compounds: $>50 \%$ of wogonoside was converted to wogonin at $2 \mathrm{~h}$. After 8-h incubation, all wogonoside had been converted to wogonin. Our data suggested that the human gut microbiome can effectively metabolize baicalin to baicalein. Due to the possibility that water soaking may structurally modify baicalin, as a control, we tested if water only (without microbiome) can convert baicalin to baicalein. Our results showed that after $8 \mathrm{~h}$ of culture, water soaking (without enzymes) did not induce such transformation. The experimental condition in this water control was different from those of our previously published report, in which botanical enzymes significantly converted baicalin to baicalein (15). Thus, our results suggested that the enteric microbiome played a critical role in metabolizing baicalin to baicalein.

To compare the antiproliferative effects between baicalin and baicalein, in addition to three human colorectal cancer cell lines (HCT-116, SW-480 and HT-29), we also used three other cancer cell lines from two common solid tumors, small cell lung cancer (NSCLC) and breast cancer (MCF-7 and MDA-MB-231). We observed that, compared to baicalin, baicalein showed much stronger antiproliferative effects on all the cancer cell lines. Basically, baicalin was not effective on NSCLC and MCF-7 cells. At $100 \mu \mathrm{M}$, baicalin did not inhibit cancer cell growth in these two cell lines. Although baicalin showed antiproliferative effects in other cancer cell lines, however, the active concentration was $>60 \mu \mathrm{M}$. On the other hand, baicalein showed very significant antiproliferative effects on all the tested cancer cell lines.

For the three human colorectal cancer cell lines, HT-29 was relatively resistant to baicalein treatment, with an $\mathrm{IC}_{50}$ of 87.3 $\mu \mathrm{M}$. Baicalein showed the most potent effects on HCT-116 cells, with an $\mathrm{IC}_{50}$ of $40.1 \mu \mathrm{M}$. Thus, we select this cell line to further validate its effects and explore anticancer metabolisms of action.

A subcutaneous HCT-116 tumor model using xenograft nude mice was established to confirm the in vitro antiproliferative effect of baicalein on colorectal cancer cells. Our data indicated that the daily administration of $30 \mathrm{mg} / \mathrm{kg}$ of baicalin inhibited the HCT-116 tumor growth at week 4. Compare to limited effects of baicalin, baicalein showed much stronger antitumor effects. After mice received $30 \mathrm{mg} / \mathrm{kg}$ of baicalein, HCT-116 tumor growth was significantly inhibited in weeks 2-4 (Fig. 3). The in vivo antitumor evaluation supported the in vitro antiproliferative effects that the enteric microbiome metabolite baicalein is an active anti-colorectal cancer compound.

Because the inhibition of cell cycle progression and induction of apoptosis are important mechanisms mediating the effects of many anticancer agents, in this study, we compared the activities of baicalin and baicalein on the cell cycle and apoptosis. Cell cycle effects of baicalin were observed only at high concentrations. At $60 \mu \mathrm{M}$, baicalin increased cell proportions in $\mathrm{G} 1$ phase and decreased $\mathrm{G} 2 / \mathrm{M}$ phase. On the other hand, at as low as $20 \mu \mathrm{M}$, baicalein showed potent effects on the cell cycle. Compared to the control, baicalein significantly dose-dependently induced HCT-116 cell cycle arrest in S phase. We previously isolated the glycoside fraction (contains baicalin and wogonoside) and aglycon fraction (contains baicalein and wogonin) from $\mathrm{SbE}$ (36). Since the aglycon fraction showed potent antiproliferative effects, we assayed cell cycle effects of aglycon fractions on HCT-116 cells. Aglycon fraction induced cell cycle arrest in both S and G2/M phases (38). Data from this study supported our previous observations, that baicalein contributed to the $\mathrm{S}$ phase arrest that was also observed by the aglycon fraction treatment.

Effects of baicalin and baicalein on HCT-116 cell apoptosis were evaluated. Baicalein markedly induced colon cancer cell apoptosis in concentrations of 20-80 $\mu \mathrm{M}$, while baicalin appeared to have positive effect at high concentration $(80 \mu \mathrm{M})$. Because caspase 3 and 9 are situated at critical points in apoptotic pathways (39), to further explore the mechanism mediating baicalein-induced apoptosis, we assayed the activities of caspase 3 and 9. At concentrations of 40 and $60 \mu \mathrm{M}$, baicalein significantly upregulated the activities of these caspases. Our docking analysis further suggested interaction sites between baicalein and caspases. Baicalein induced apoptosis may be mediated by direct physical interactions with these targets. Our results suggest that the cancer cell growth inhibitory effect of baicalein was predominantly mediated by induction of apoptosis.

In conclusion, using the human enteric microbiome, the biotransformation and metabolic profile of S. baicalensis flavonoids was determined. Baicalin can be easily metabolized to baicalein. Using a panel of human cancer cell lines, we tested the antiproliferative effects of baicalin and baicalein. As a major parent compound from $S$. baicalensis, baicalin showed limited antiproliferative effects on some of these cell lines. Baicalein, however, showed significant antiproliferative effects in all the tested cancer cell lines, especially on HCT-116 human colorectal cancer cells. In vivo antitumor results supported our in vitro data. We demonstrated that baicalein exerts potent $\mathrm{S}$ phase cell cycle arrest and pro-apoptotic effects in HCT-116 cells. Baicalein upregulated the expression of caspase 3 and 9. Baicalein-induced apoptosis could be through direct physical interactions with these apoptotic regulators. Data from this study suggested that baicalein is a potent anticancer metabolite derived from $S$. baicalensis. Enteric microbiota play a key role in the colon cancer chemoprevention of S. baicalensis.

\section{Acknowledgements}

This study was supported in part by the NIH/NCCAM grants K01 AT005362 and P01 AT004418.

\section{References}

1. Torre LA, Bray F, Siegel RL, Ferlay J, Lortet-Tieulent J and Jemal A: Global cancer statistics, 2012. CA Cancer J Clin 65: 87-108, 2015 
2. Siegel RL, Miller KD and Jemal A: Cancer statistics, 2015. CA Cancer J Clin 65: 5-29, 2015.

3. Gray R, Barnwell J, McConkey C, Hills RK, Williams NS and Kerr DJ; Quasar Collaborative Group: Adjuvant chemotherapy versus observation in patients with colorectal cancer: A randomised study. Lancet 370: 2020-2029, 2007.

4. Ernst E: The role of complementary and alternative medicine in cancer. Lancet Oncol 1: 176-180, 2000.

5. Sampson W: Natural products and cancer. In: A Textbook of Complementary and Alternative Medicine. 2nd edition. Yuan CS, Bieber EJ and Bauer BA (eds). Parthenon/CRC, London, pp645-654, 2006.

6. Cragg GM, Grothaus PG and Newman DJ: Impact of natural products on developing new anti-cancer agents. Chem Rev 109: 3012-3043, 2009.

7. Butler MS, Robertson AA and Cooper MA: Natural product and natural product derived drugs in clinical trials. Nat Prod Rep 31: 1612-1661, 2014.

8. Wang CY, Bai XY and Wang CH: Traditional Chinese medicine: A treasured natural resource of anticancer drug research and development. Am J Chin Med 42: 543-559, 2014.

9. Chen R, Zhang J, Hu Y, Wang S, Chen M and Wang Y: Potential antineoplastic effects of Aloe-emodin: A comprehensive review. Am J Chin Med 42: 275-288, 2014.

10. Kim EH, Shim B, Kang S, Jeong G, Lee JS, Yu YB and Chun M: Anti-inflammatory effects of Scutellaria baicalensis extract via suppression of immune modulators and MAP kinase signaling molecules. J Ethnopharmacol 126: 320-331, 2009.

11. Arweiler NB, Pergola G, Kuenz J, Hellwig E, Sculean A and Auschill TM: Clinical and antibacterial effect of an anti-inflammatory toothpaste formulation with Scutellaria baicalensis extract on experimental gingivitis. Clin Oral Investig 15 909-913, 2011

12. Li-Weber M: New therapeutic aspects of flavones: The anticancer properties of Scutellaria and its main active constituents Wogonin, Baicalein and Baicalin. Cancer Treat Rev 35: 57-68, 2009.

13. Chen HM, Liou SF, Hsu JH, Chen TJ, Cheng TL, Chiu CC and Yeh JL: Baicalein inhibits HMGB1 release and MMP-2/-9 expression in lipopolysaccharide-induced cardiac hypertrophy. Am J Chin Med 42: 785-797, 2014

14. Li C, Zhou L, Lin G and Zuo Z: Contents of major bioactive flavones in proprietary traditional Chinese medicine products and reference herb of radix Scutellariae. J Pharm Biomed Anal 50: 298-306, 2009.

15. Yu C, Qu F, Mao Y, Li D, Zhen Z, Nass R, Calway T, Wang Y, Yuan CS and Wang CZ: Different extraction pretreatments significantly change the flavonoid contents of Scutellaria baicalensis. Pharm Biol 51: 1228-1235, 2013.

16. Shi R, Zhou H, Liu Z, Ma Y, Wang T, Liu Y and Wang C: Influence of coptis Chinensis on pharmacokinetics of flavonoids after oral administration of radix Scutellariae in rats. Biopharm Drug Dispos 30: 398-410, 2009.

17. Hasegawa $\mathrm{H}$ : Proof of the mysterious efficacy of ginseng: Basic and clinical trials: metabolic activation of ginsenoside: Deglycosylation by intestinal bacteria and esterification with fatty acid. J Pharmacol Sci 95: 153-157, 2004.

18. Liu H, Yang J, Du F, Gao X, Ma X, Huang Y, Xu F, Niu W, Wang F, Mao Y, et al: Absorption and disposition of ginsenosides after oral administration of Panax notoginseng extract to rats. Drug Metab Dispos 37: 2290-2298, 2009.

19. Tawab MA, Bahr U, Karas M, Wurglics M and SchubertZsilavecz M: Degradation of ginsenosides in humans after oral administration. Drug Metab Dispos 31: 1065-1071, 2003.

20. Yu C, Zhang Z, Zhang H, Zhen Z, Calway T, Wang Y, Yuan CS and Wang CZ: Pretreatment of baicalin and wogonoside with glycoside hydrolase: A promising approach to enhance anticancer potential. Oncol Rep 30: 2411-2418, 2013.

21. Wang CZ, Du GJ, Zhang Z, Wen XD, Calway T, Zhen Z, Musch MW, Bissonnette M, Chang EB and Yuan CS: Ginsenoside compound $\mathrm{K}$, not $\mathrm{Rb} 1$, possesses potential chemopreventive activities in human colorectal cancer. Int J Oncol 40: 1970-1976, 2012.

22. Gao JL, Lv GY, He BC, Zhang BQ, Zhang H, Wang N, Wang CZ, $\mathrm{Du}$ W, Yuan CS and He TC: Ginseng saponin metabolite 20(S)-protopanaxadiol inhibits tumor growth by targeting multiple cancer signaling pathways. Oncol Rep 30: 292-298, 2013.
23. Gong WY, Wu JF, Liu BJ, Zhang HY, Cao YX, Sun J, Lv YB, Wu X and Dong JC: Flavonoid components in Scutellaria baicalensis inhibit nicotine-induced proliferation, metastasis and lung cancer-associated inflammation in vitro. Int J Oncol 44 1561-1570, 2014.

24. Lin C, Tsai SC, Tseng MT, Peng SF, Kuo SC, Lin MW, Hsu YM, Lee MR, Amagaya S, Huang WW, et al: AKT serine/threonine protein kinase modulates baicalin-triggered autophagy in human bladder cancer T24 cells. Int J Oncol 42: 993-1000, 2013.

25. Wan JY, Liu P, Wang HY, Qi LW, Wang CZ, Li P and Yuan CS: Biotransformation and metabolic profile of American ginseng saponins with human intestinal microflora by liquid chromatography quadrupole time-of-flight mass spectrometry. J Chromatogr A 1286: 83-92, 2013.

26. Chen WC, Kuo TH, Tzeng YS and Tsai YC: Baicalin induces apoptosis in SW620 human colorectal carcinoma cells in vitro and suppresses tumor growth in vivo. Molecules 17: 3844-3857, 2012.

27. Kim SJ, Kim HJ, Kim HR, Lee SH, Cho SD, Choi CS, Nam JS and Jung JY: Antitumor actions of baicalein and wogonin in HT-29 human colorectal cancer cells. Mol Med Rep 6: 1443-1449, 2012

28. Xu Z, Wu G, Wei X, Chen X, Wang Y and Chen L: Celastrol induced DNA damage, cell cycle arrest, and apoptosis in human rheumatoid fibroblast-like synovial cells. Am J Chin Med 41: 615-628, 2013.

29. Sai K, Li WY, Chen YS, Wang J, Guan S, Yang QY, Guo CC, Mou YG, Li WP and Chen ZP: Triptolide synergistically enhances temozolomide-induced apoptosis and potentiates inhibition of NF- $x \mathrm{~B}$ signaling in glioma initiating cells. Am J Chin Med 42: 485-503, 2014.

30. Jain AN: Morphological similarity: A 3D molecular similarity method correlated with protein-ligand recognition. J Comput Aided Mol Des 14: 199-213, 2000.

31. Giganti D, Guillemain H, Spadoni JL, Nilges M, Zagury JF and Montes M: Comparative evaluation of 3D virtual ligand screening methods: Impact of the molecular alignment on enrichment. J Chem Inf Model 50: 992-1004, 2010.

32. Kim R, Tanabe K, Uchida Y,Emi M, Inoue H and Toge T: Current status of the molecular mechanisms of anticancer drug-induced apoptosis. The contribution of molecular-level analysis to cancer chemotherapy. Cancer Chemother Pharmacol 50: 343-352, 2002.

33. Hanna RK and Soper JT: The role of surgery and radiation therapy in the management of gestational trophoblastic disease. Oncologist 15: 593-600, 2010.

34. Russo R, Corasaniti MT, Bagetta G and Morrone LA: Exploitation of cytotoxicity of some essential oils for translation in cancer therapy. Evid Based Complement Alternat Med 2015: 397821, 2015.

35. Wang CZ, Li XL, Wang QF, Mehendale SR, Fishbein AB, Han AH, Sun S and Yuan CS: The mitochondrial pathway is involved in American ginseng-induced apoptosis of SW-480 colon cancer cells. Oncol Rep 21: 577-584, 2009.

36. Wang CZ, Li XL, Wang QF, Mehendale SR and Yuan CS: Selective fraction of Scutellaria baicalensis and its chemopreventive effects on MCF-7 human breast cancer cells. Phytomedicine 17: 63-68, 2010.

37. Zhang J, Park HS, Kim JA, Hong GE, Nagappan A, Park KI and Kim GS: Flavonoids identified from Korean Scutellaria baicalensis induce apoptosis by ROS generation and caspase activation on human fibrosarcoma cells. Am J Chin Med 42: 465-483, 2014.

38. Wang CZ, Calway TD, Wen XD, Smith J, Yu C, Wang Y, Mehendale SR and Yuan CS: Hydrophobic flavonoids from Scutellaria baicalensis induce colorectal cancer cell apoptosis through a mitochondrial-mediated pathway. Int J Oncol 42: 10181026, 2013.

39. Ismail B, Ghezali L, Gueye R, Limami Y, Pouget C, Leger DY, Martin F, Beneytout JL, Duroux JL, Diab-Assaf M, et al: Novel methylsulfonyl chalcones as potential antiproliferative drugs for human prostate cancer: Involvement of the intrinsic pathway of apoptosis. Int J Oncol 43: 1160-1168, 2013. 